* Encoding: UTF-8.

\title{
FACTOR
}

/VARIABLES vf nvf tol acc

/MISSING MEANSUB

/ANALYSIS vf nvf tol acc

/PRINT UNIVARIATE INITIAL CORRELATION SIG KMO EXTRACTION ROTATION FSCORE

/CRITERIA MINEIGEN(1) ITERATE(25)

/EXTRACTION PC

/CRITERIA ITERATE(25)

/ROTATION VARIMAX

/SAVE REG(ALL)

/METHOD=CORRELATION.

\section{Factor Analysis}

Descriptive Statistics

\begin{tabular}{lrrrr}
\hline & Mean & Std. Deviation $^{\mathrm{a}}$ & Analysis N $^{a}$ & Missing N \\
\hline Vertebral fractures & .5000 & .27227 & 15 & 0 \\
Nonvertebral fractures & .5000 & .24203 & 15 & 0 \\
Tolerability & .5000 & .22730 & 15 & 1 \\
Acceptability & .5000 & .23268 & 15 & 0 \\
\hline
\end{tabular}

a. For each variable, missing values are replaced with the variable mean.

Correlation Matrix

\begin{tabular}{llrrrr}
\hline & & $\begin{array}{c}\text { Vertebral } \\
\text { fractures }\end{array}$ & $\begin{array}{c}\text { Nonvertebral } \\
\text { fractures }\end{array}$ & Tolerability & Acceptability \\
\hline \multirow{2}{*}{ Correlation } & Vertebral fractures & 1.000 & .796 & -.480 & -.401 \\
& Nonvertebral fractures & .796 & 1.000 & -.160 & -.283 \\
& Tolerability & -.480 & -.160 & 1.000 & .545 \\
& Acceptability & -.401 & -.283 & .545 & 1.000 \\
\hline \multirow{2}{*}{ Sig. (1-tailed) } & Vertebral fractures & & .000 & .035 & .069 \\
& Nonvertebral fractures & .000 & & .285 & .153 \\
& Tolerability & .035 & .285 & & .022 \\
\hline
\end{tabular}


KMO and Bartlett's Test

\begin{tabular}{|c|c|c|}
\hline \multicolumn{2}{|c|}{ Kaiser-Meyer-Olkin Measure of Sampling Adequacy. } & .509 \\
\hline \multirow[t]{3}{*}{ Bartlett's Test of Sphericity } & Approx. Chi-Square & 20.270 \\
\hline & df & 6 \\
\hline & Sig. & .002 \\
\hline
\end{tabular}

Communalities

\begin{tabular}{lcc}
\hline & Initial & Extraction \\
\hline Vertebral fractures & 1.000 & .906 \\
Nonvertebral fractures & 1.000 & .940 \\
Tolerability & 1.000 & .808 \\
Acceptability & 1.000 & .733 \\
\hline
\end{tabular}

Extraction Method: Principal Component Analysis.

Total Variance Explained

\begin{tabular}{lcrrrr}
\hline & \multicolumn{3}{c}{ Initial Eigenvalues } & \multicolumn{2}{c}{ Extraction Sums of Squared .. } \\
Component & Total & \% of Variance & Cumulative \% & Total & \% of Variance \\
\hline 1 & 2.353 & 58.837 & 58.837 & 2.353 & 58.837 \\
2 & 1.033 & 25.833 & 84.670 & 1.033 & 25.833 \\
3 & .482 & 12.045 & 96.715 & & \\
4 & .131 & 3.285 & 100.000 & & \\
\hline
\end{tabular}

Total Variance Explained

\begin{tabular}{lrrrr}
\hline & \multicolumn{3}{c}{ Extraction Sums ... } & \multicolumn{3}{c}{ Rotation Sums of Squared Loadings } \\
Component & Cumulative \% & Total & \% of Variance & Cumulative \% \\
\hline 1 & 58.837 & 1.764 & 44.101 & 44.101 \\
2 & 84.670 & 1.623 & 40.569 & 84.670 \\
3 & & & & \\
4 & & & & \\
\hline
\end{tabular}

Extraction Method: Principal Component Analysis. 
Component Matrix $^{a}$

\begin{tabular}{lll}
\hline & \multicolumn{2}{c}{ Component } \\
& 1 & 2 \\
\hline Vertebral fractures & .899 & .314 \\
Nonvertebral fractures & .757 & .605 \\
Tolerability & -.691 & .574 \\
Acceptability & -.703 & .489 \\
\hline
\end{tabular}

Extraction Method: Principal Component Analysis.

a. 2 components extracted.

Rotated Component Matrix ${ }^{a}$

\begin{tabular}{lcc}
\hline & \multicolumn{2}{c}{ Component } \\
& 1 & 2 \\
\hline Vertebral fractures & .879 & -.367 \\
Nonvertebral fractures & .968 & -.056 \\
Tolerability & -.131 & .889 \\
Acceptability & -.197 & .833 \\
\hline
\end{tabular}

Extraction Method: Principal Component Analysis.

Rotation Method: Varimax with Kaiser Normalization.

a. Rotation converged in 3 iterations.

\section{Component Transformation \\ Matrix}

\begin{tabular}{lll}
\hline Component & 1 & 2 \\
\hline 1 & .744 & -.668 \\
2 & .668 & .744 \\
\hline
\end{tabular}

Extraction Method: Principal Component Analysis.

Rotation Method: Varimax with

Kaiser Normalization. 


\section{Component Score Coefficient Matrix}

\begin{tabular}{lll} 
& \multicolumn{2}{c}{ Component } \\
& 1 & 2 \\
\hline Vertebral fractures & .487 & -.029 \\
Nonvertebral fractures & .631 & .221 \\
Tolerability & .153 & .610 \\
Acceptability & .094 & .551 \\
\hline
\end{tabular}

Extraction Method: Principal Component Analysis.

Rotation Method: Varimax with Kaiser

Normalization.

Component Scores.

\section{Component Score Covariance}

\section{Matrix}

\begin{tabular}{lrr}
\hline Component & 1 & \multicolumn{1}{c}{2} \\
\hline 1 & 1.000 & .000 \\
2 & .000 & 1.000 \\
\hline
\end{tabular}

Extraction Method: Principal

Component Analysis.

Rotation Method: Varimax with

Kaiser Normalization.

Component Scores. 